uniquely as a product of a finite number of prime differential ideals. The consistency of the axioms is easily shown. If we define differentiation in the ring $C(x)$, obtained by adjoining $x$ to the field of the rational numbers, in any way so as to leave it closed, it may be shown that Axioms I-IV are always satisfied. In $C(x, y)$ differentiation may be defined in such way that the statement as above still holds. This is of interest because every ordinary ideal in $C(x, y)$ may not be expressed uniquely as a product of a finite number of prime ideals.

Hofstra College

\title{
ON THE ITERATION OF LINEAR HOMOGENEOUS TRANSFORMATIONS
}

\section{ARNOLD DRESDEN}

1. Statement of problem. The question which this note tries to answer is that of determining under what conditions on the matrix $\left(a_{i j}\right)$, $(i, j=1, \cdots, n)$, the $n$-fold multiple sequence of complex numbers $x_{k}, x_{k}^{\prime}, \cdots, x_{k}^{(m)}, \cdots(k=1,2, \cdots, n)$ obtained by iteration of the linear homogeneous transformation $x_{k}^{\prime}=a_{k j} x_{j}$ will converge for every initial set $x=\left(x_{1}, x_{2}, \cdots, x_{n}\right)$. Convergence is to be understood in the sense that there exists a set $X_{1}, X_{2}, \cdots, X_{n}$ such that, for $k=1,2, \cdots, n, x_{k}^{(m)} \rightarrow X_{k}$, as $m \rightarrow \infty$.

2. Jordan normal form. We begin by recalling that a matrix $A=\left(a_{i j}\right)$ with complex elements is similar to its Jordan normal form $J_{0}$. This means that there exists a unimodular matrix $P$, such that $A=P^{-1} J_{0} P$ and $J_{0}=P A P^{-1}$, where $J_{0}$ is the direct sum of Jordan matrices $J_{1}, \cdots, J_{N}$. To each elementary divisor $\left(\lambda-\lambda_{\rho}\right)^{e_{\rho}}$ of the characteristic matrix $\lambda I-A(\rho=1,2, \cdots, N)$ and $e_{1}+e_{2}+\cdots+e_{N}$ $=n$, corresponds a Jordan matrix $J_{\rho}$. If $e_{\rho}>1$, then $J_{\rho}$ has zero elements everywhere, except in the principal diagonal, all of whose elements are $\lambda_{\rho}$, and in the diagonal immediately below the principal diagonal, all of whose elements are $1 .{ }^{1}$ If $e_{\rho}=1$, then $J_{\rho}$ consists of the single element $\lambda_{\rho}$.

It follows that any integral power of $J_{0}$ is the direct sum of the same powers of the Jordan matrices $J_{\rho}$. Let us now denote by $J$ an arbitrary Jordan matrix of order $n>1$,

Received by the editors August 25, 1941.

${ }^{1}$ See, for example, MacDuffee, Introduction to Abstract Algebra, p. 241. 


$$
J=\left(\begin{array}{cccccccc}
\lambda & 0 & 0 & 0 & \ldots & . & . & 0 \\
1 & \lambda & 0 & 0 & \ldots & . & . & 0 \\
0 & 1 & \lambda & 0 & \ldots & . & . & 0 \\
\cdot & . & . & . & \ldots & . & . & . \\
0 & . & . & . & \cdots & 0 & \lambda & 0 \\
0 & . & . & . & \ldots & 0 & 1 & \lambda
\end{array}\right) .
$$

It is then easily seen that $J^{2}$ is a matrix of order $n$, whose first column is $\lambda^{2}, 2 \lambda, 1,0, \cdots, 0$, and whose second column is obtained from the first column by shifting the elements one place downward, as far as the length permits, and placing 0 in the first place; the third column is obtained from the second in similar manner; the last column is $00 \cdots 0 \lambda^{2}$. A simple induction enables us to prove that, for any positive integer $h$, the matrix $J^{h}$ is of order $n$, that its first column consists of the successive terms in the binomial expansion of $(\lambda+1)^{h}$, followed by zeros, provided $n \geqq h+1$ and otherwise of as many of these terms as the length of the column permits, that each of its columns after the first is obtained from the one to the left of it by shifting the elements one place downward and filling the open position above with a zero, and that its last column is $000 \cdots 0 \lambda^{h}$. If $J$ is a Jordan matrix of order 1 , consisting of the single element $\lambda$ then $J^{n}$ consists of the single element $\lambda^{n}$.

3. Reduction of problem; solution. Returning now to our linear homogeneous transformation, we write it in the form

$$
x^{\prime}=x A \text {, }
$$

where $x$ and $x^{\prime}$ stand for the row-vectors $\left(x_{1}, x_{2}, \cdots, x_{n}\right)$ and $\left(x_{1}^{\prime}, x_{2}^{\prime}, \cdots, x_{n}^{\prime}\right)$, respectively. By means of the unimodular matrix $P$, we map the space of the row-vectors $x$ on the space of the rowvectors $y: x=y P, \quad y=x P^{-1}$. This mapping carries the transformation (1) into a transformation in the $y$-space:

$$
y^{\prime}=x^{\prime} P^{-1}=x A P^{-1}=y P A P^{-1}=y J_{0} .
$$

It follows from the mapping that the vectors $x^{(m)}$ will converge, in the sense described in $\S 1$, if and only if the vectors $y^{(m)}$ converge. But $y^{(m)}=y^{(m-1)} J_{0}=\cdots=y_{0} J_{0}^{m}=y\left(J_{1}^{m}+J_{2}^{m}+\cdots+J_{N}^{m}\right)$. We obtain therefore the following form for the $m$ th iterate of the transformation (2) in terms of the roots $\lambda_{\rho}$ of the characteristic equation $\left|a_{i j}-\lambda \delta_{i j}\right|=0$ and the exponents $e_{\rho}$ of the corresponding elementary divisors $\left(\lambda-\lambda_{\rho}\right)^{e_{\rho}}$ of $\lambda I-A$, provided every $e_{\rho}>1$.

$$
\begin{aligned}
& y_{1}^{(m)}=\lambda_{1}^{m} y_{1}+C_{m, 1} \lambda_{1}^{m-1} y_{2}+\cdots+C_{m, e_{1}-1} \lambda_{1}^{m-e_{1}+1} y_{e_{1}}, \\
& y_{2}^{(m)}=\lambda_{1}^{m} y_{2}+C_{m, 1} \lambda_{1}^{m-1} y_{3}+\cdots+C_{m, e_{1}-2} \lambda_{1}^{m-e_{1}+2} y_{e_{1}},
\end{aligned}
$$




$$
\begin{aligned}
& y_{e_{1}}^{(m)}=\lambda_{1}^{m} y_{e_{1}} \text {, } \\
& y_{e_{1}+1}^{(m)}=\lambda_{2}^{m} y_{e_{1}+1}+C_{m, 1} \lambda_{2}^{m-1} y_{e_{1}+2}+\cdots+C_{m, e_{2}-1} \lambda_{2}^{m-e_{2}+1} y_{e_{1}+e_{2}} \text {, } \\
& (m) \\
& y_{e_{1}+1}= \\
& \lambda_{2}^{m} y_{e_{1}+2}+\cdots+C_{m, e_{2}-2} \lambda_{2}^{m-e_{2}+2} y_{e_{1}+e_{2}} \text {, } \\
& y_{e_{1}+e_{2}}^{(m)}=\lambda_{2}^{m} y_{e_{1}+e_{2}} \\
& y_{n}^{(m)}=\lambda_{N}^{m} y_{n} \text {. }
\end{aligned}
$$

This form makes it evident that the set $y_{k}^{(m)}$ will converge as $m$ increases indefinitely, if and only if $\left|\lambda_{\rho}\right|<1$, for $\rho=1, \cdots, N$. If, on the other hand, all the elementary divisors involving a root $\lambda_{\rho}$ are linear, then convergence will occur also if $\lambda_{\rho}=1$. We have therefore obtained the following answer to our question:

THEOREM. The iteration of the linear homogeneous transformation $x_{k}^{\prime}=a_{k} x_{j}(k, j=1,2, \cdots, n)$ will converge for every initial set $x_{k}$ if and only if all the roots of the characteristic equation of the matrix $\left(a_{i j}\right)$ are less than unity in absolute value, except that the roots, which are involved only in linear elementary divisors, may be equal to unity. ${ }^{2}$

4. Remark. It is clear that it is possible for some of the variables $y_{k}^{(m)}$ to converge to limits while others do not converge; for example, the iteration of the transformation $y_{1}^{\prime}=1 / 2 y_{1}, y_{2}^{\prime}=y_{2}+y_{3}, y_{3}^{\prime}=y_{3}+y_{4}$, $y_{4}^{\prime}=y_{4}$ will obviously yield a convergent sequence for $y_{1}^{(m)}$ and for $y_{4}^{(m)}$, but not for $y_{2}^{(m)}$, if we start with the initial set $y_{1}=a, y_{3}=b, y_{2}=y_{4}=0$. In this case the roots of the characteristic equation are $1 / 2$ and 1 , with multiplicities 1 and 3 , respectively. It may be worth while to determine, in the general case of the linear homogeneous transformation, which variables do and which do not converge upon iteration, in case some, but not all the roots of the characteristic roots of the matrix of the transformation are less than unity in absolute value.

\section{Swarthmore College}

2 In an earlier incomplete formulation of this theorem, the exceptional character of the linear elementary divisors was overlooked. The author is indebted for the detection of this error to Professor W. T. Reid, who called attention to the fact that convergence would take place if $A$ were an idempotent matrix. In this connection it may be of interest to observe that for a matrix which satisfies an equation of the form $A^{r+1}=A^{r}, r \geqq 1, a l l$ the elementary divisors of $\lambda I-A$ are linear. For, on the one hand, its Jordan normal form would have to satisfy the same relation, while it follows from our discussion that this can not be the case if $\lambda I-A$ has a non-linear elementary divisor. 